\title{
Therapeutic effects of NogoA vaccine and olfactory ensheathing glial cell implantation on acute spinal cord injury
}

This article was published in the following Dove Press journal:

Journal of Neurorestoratology

25 October 2013

Number of times this article has been viewed

\author{
Zhicheng Zhang \\ Fang Li \\ Tiansheng Sun \\ Dajiang Ren \\ Xiumei Liu
}

PLA Institute of Orthopedics, Beijing Army General Hospital, Beijing, People's Republic of China

Correspondence: Tiansheng Sun PLA Institute of Orthopedics, Beijing Army General Hospital, 5 Nanmencang, Dongcheng District, Beijing, People's Republic of China Fax +86 I0 84042490

Email suntiansheng-@163.com
Background: Many previous studies have focused on the effects of IN-1, a monoclonal antibody that neutralizes Nogo (a neurite growth inhibitory protein), on neurologic regeneration in spinal cord injury (SCI). However, safety problems and the short half-life of the exogenous antibody are still problematic. In the present study, the NogoA polypeptide was used as an antigen to make a therapeutic NogoA vaccine. Rats were immunized with this vaccine and were able to secrete the polyclonal antibody before SCI. The antibody can block NogoA within the injured spinal cord when the antibody gains access to the spinal cord due to a compromised blood-spinal cord barrier. Olfactory ensheathing glial cell transplantation has been used in a spinal cord contusion model to promote the recovery of SCI. The present study was designed to verify the efficacy and safety of NogoA polypeptide vaccine, the effects of immunotherapy with this vaccine, and the synergistic effects of the vaccine and olfactory ensheathing glial cells in repair of SCI.

Methods: A 13-polypeptide fragment of NogoA was synthesized. This fragment was then coupled with keyhole limpet hemocyanin to improve the immunogenicity of the polypeptide vaccine. Immunization via injection into the abdominal cavity was performed in rats before SCI. The serum antibody level and ability of the vaccine to bind with Nogo were detected by enzymelinked immunosorbent assay. The safety of the vaccine was evaluated according to the incidence and severity of experimental autoimmune encephalomyelitis. Olfactory ensheathing glia cells were obtained, purified, and subsequently implanted into a Wistar rat model of thoracic spinal cord contusion injury. The rats were divided into four groups, ie, an SCI model group, an olfactory ensheathing glia group, a vaccine group, and a combined treatment group. Animal behavior, histopathology, and axonal regeneration were compared between the four treatment groups.

Results: The antibody against the polypeptide was detected in rat serum by enzyme-linked immunosorbent assay. Experimental autoimmune encephalomyelitis was not found in the immunized rats. Three months after injury, Basso, Beattie, Bresnahan scores and nerve fiber counts in biotinylated dextran amine nerve tracing studies were significantly better in the combined treatment group than in the other groups.

Conclusion: The polypeptide NogoA vaccine can stimulate the humoral immune system to produce antibodies against the NogoA polypeptide. The binding reaction between the antibody and antigen was shown in ex vivo experiments. No evidence was found to suggest a relationship between NogoA vaccination and increased risk of experimental autoimmune encephalomyelitis. The combined strategy of olfactory ensheathing glial cell implantation and NogoA vaccination may promote regeneration of axons and functional recovery in the spinal cord contusion injury model. This study may provide a new strategy for combining modalities to enhance axonal regeneration and a better balance of the CNS microenvironment after SCI.

Keywords: spinal cord injury, immunotherapy, cell transplantation, olfactory ensheathing glial cells, NogoA, vaccine 


\section{Introduction}

Central nervous system (CNS) axonal injury in higher vertebrates is usually difficult to reverse, but axons in the peripheral nervous system (PNS) can regenerate rapidly. Benfey and Aguayo ${ }^{1}$ found that CNS axons can regenerate in PNS tissue, but PNS axons cannot regenerate in CNS tissue. This means that the difficulty in reversing CNS injury does not result from the nature of the neurons but from the local regenerative microenvironment. The main problems are that the CNS microenvironment is not conducive to axonal regeneration and the regenerative ability of CNS neurons is low. The microenvironment includes two aspects, ie, promoting factors and inhibiting factors. Neurotrophic factors are the main promoters required for axonal regeneration, whereas neurite growth inhibitory proteins account for the main inhibitory effects in axonal regeneration. Horner and $\mathrm{Gage}^{2}$ pointed out that axonal regeneration after CNS injury depends mainly on a delicate coordination of promotion factors and inhibitory factors in the microenvironment, meaning that we should eliminate inhibiting factors while increasing supportive factors as far as possible.

In recent decades, much effort has been devoted to exploring repair strategies in the hostile microenvironment of the CNS to provide injured spinal cord axons with a supportive environment. Some of these therapeutic approaches include tissue implantation, administration of neurotrophic factors, gene therapy, block of myelin inhibition, and cell transplantation. Although the efficacy of these experimental methods has been verified in animal studies, it would take a long time for such measures to reach the bedside. Spinal cord injury (SCI) has attracted a lot of research attention with the same aim, ie, to find a way to promote axonal regeneration, remyelination, and functional recovery, by using simple, safe, effective, and viable procedures. ${ }^{3}$ Olfactory ensheathing glial cell (OEG) transplantation has emerged as a very promising experimental therapy to promote repair of SCI. ${ }^{4}$

OEGs have several roles in the repair of SCI. First, they can contribute to improvement of the local microenvironment of SCI. In vitro and in vivo studies have found that OEG secretes many neurotrophins, including nerve growth factor, brain-derived neurotrophic factor, neurotrophin-3 (NT-3), neurotrophin-4, platelet-derived growth factor, neuropeptide $\mathrm{Y}$, and S100 membrane molecules related to cell adhesion and axonal growth. Second, OEGs have also been demonstrated to be efficient in promoting regeneration of axons after SCI. Third, OEGs had the ability to elongate axons through the glia scar. ${ }^{5}$

However, according to the existing evidence, the longterm functional recovery resulting from OEG transplantation alone is still not satisfactory. In order to enhance the repair function of OEGs, these cells has been modified by many neurotrophic factor-related genes. Our study ${ }^{6}$ of NT-3 genemodified OEGs in the repair of rat SCI shows that the Basso, Beattie, Bresnahan (BBB) score ${ }^{7}$ was higher than for OEGs alone, but the score of NT-3 modified OEGs group eventually formed a plateau phase of merely 13 points. Another study of brain-derived neurotrophic factor gene-modified OEG transplantation in the treatment of SCI had similar results. In fact, it was found that the damaged area still had high expression of the axon inhibitory protein, NogoA, after OEG transplantation. ${ }^{8}$ Hence, we speculated that the reason for unsatisfactory functional recovery from SCI was that OEGs, although modified by many neurotrophic factors, cannot neutralize myelin-derived axon-suppressive proteins. This procedure cannot completely eliminate inhibitory factors or promote coordination of the CNS microenvironment.

There are two main inhibitory factors in the microenvironment of the injured spinal cord. ${ }^{9}$ The first is the glial scar. ${ }^{10}$ Undoubtedly, the glial scar can directly block regeneration, but it takes weeks to be fully formed. The second is myelin. Myelin is an important component of the adult CNS microenvironment that acts to prevent axon regeneration. Three inhibitors from myelin that have been identified are Nogo, myelin-associated glycoprotein, and oligodendrocyte myelin glycoprotein. Nogo plays a very important role in inhibition of axonal regeneration. Nogo is a member of the reticulon family and occurs in three forms, ie, NogoA, NogoB, and NogoC. All three isoforms of Nogo share a 66-amino acid residue in the luminal/extracellular domain (Nogo-66), which inhibits axonal extension and spreading of fibroblasts. ${ }^{11}$

In order to neutralize Nogo-66, Caroni and Schwab ${ }^{12}$ used a monoclonal antibody against this myeloid-derived axonal regeneration inhibitory protein. That antibody, termed IN-1, was shown to allow axons to grow. ${ }^{13}$ A number of axons were shown to extend relatively long distances and, in some cases, functional recovery occurred in a rat model of SCI. Further, application of IN-1 with NT-3 or brain-derived neurotrophic factor may increase the effect of repair. Jensen et al ${ }^{14}$ reported that not only in damaged area of spinal cord but also in the adjacent area of injured spinal cord, the blood-spinal cord barrier was destroyed and opened. This may be because of activation of microglia and macrophages. This type of selective opening of the blood-spinal cord barrier in the region adjacent to the damaged spinal cord may be beneficial for entry of antibodies into the SCI area. This makes research and treatment of SCI using a vaccine possible. Huang et a ${ }^{15}$ reported on mice that had been immunized with purified 
myelin and incomplete Freund's adjuvant before SCI. In their study, the polyclonal antibody blocked the inhibitory effects of myelin. Adult mice immunized in this manner showed extensive regeneration of large numbers of axons in the corticospinal tracts after dorsal hemisection of the spinal cord. There was functional recovery of hind limb motor activity without a harmful cellular immune response or experimental autoimmune encephalomyelitis.

In order to prevent experimental autoimmune encephalomyelitis induced by full sequence Nogo-66 as an antigen, we developed a Nogo-13 polypeptide, which is the main functional segment, as an antigenic determinant for a vaccine to immunize rats. The IN-1 antibody was secreted into the blood of the immunized rats pre-SCI. This antibody may neutralize the inhibitory effects of NogoA immediately after SCI via the damaged blood-spinal cord barrier, thereby acting as a "therapeutic vaccine".

Therefore, in this study, we coupled Nogo-13 polypeptide, which is the main functional segment of Nogo-66, and keyhole limpet hemocyanin $(\mathrm{KLH})$ protein to form a vaccine. The rats were immunized with the vaccine and secreted the polyclonal antibody against Nogo-66. When the contusion model of SCI was created, the blood-spinal cord barrier was opened and the antibody in the blood could gain access to the injured area and have a reparative effect. At the same time, OEGs were implanted into the injured spinal cord where they could cross the glial scar, secrete various neurotrophic factors, and induce axonal regeneration. These measures were intended to achieve a delicate coordination of supportive factors and inhibitory factors in the microenvironment and provide an optimal microenvironment for axonal regeneration after SCI.

\section{Materials and methods Preparation, purification, and evaluation of Nogo vaccine}

\section{Synthesis and purification}

A combination of Nogo-13 and KLH:Nogo-13 (amino acid sequence YDKPFEQAWEVKD) was created by solid phase synthesis and purified by high-performance liquid chromatography. Purity was at least 95\%. Nogo-13 and KLH were coupled using the bis-diazotized benzidine method in an ice bath for 1.5 hours, then placed in a dialysis bag and dialyzed in physiologic saline for 48 hours. The vaccine was purified with dextran (Sephadex G250) at pH 7.2. Using an ultraviolet detector at a level of $280 \mathrm{~nm}$, the first peak detected was the polypeptide of KLH and Nogo-13. The eluent containing polypeptide was collected and measured. The vaccine was kept at $-20^{\circ} \mathrm{C}$ with concentration of $5 \mathrm{mg} / \mathrm{mL}$.

\section{Animal immunization}

Three-week-old Wistar rats $(n=60)$ were randomly allocated into groups of 20 rats each to receive NogoA vaccine immunization (group $\alpha$ ), complete Freund's adjuvant + incomplete Freund's adjuvant immunization (group $\beta$ ), or KLH immunization (group $\gamma$ ).

In group $\alpha$, the first immunization was performed with emulsified complete Freund's adjuvant + NogoA vaccine when the rats were aged 21 days. When aged 35 days, the second immunization was performed using emulsified incomplete Freund's adjuvant + NogoA vaccine. Two weeks later, the third immunization was performed using emulsified incomplete Freund's adjuvant + NogoA vaccine. In group $\beta$, complete Freund's adjuvant, incomplete Freund's adjuvant, and incomplete Freund's adjuvant were injected in the abovementioned sequence at the same dose and at the same times. Rats in group $\gamma$ were immunized with $0.5 \mathrm{mg}$ of $\mathrm{KLH}$ at the same time points. All rats were immunized by intraperitoneal injection. The NogoA vaccine $(0.5 \mathrm{mg})$ and complete or incomplete Freund's adjuvant were well mixed and emulsified at a ratio of $1: 1$.

\section{Evaluation of immunologic effects of NogoA vaccine}

Serum samples were collected 14 days after the third immunization. Rat tail blood ( $1 \mathrm{~mL}$ ) was maintained at room temperature for 2 hours, in a water bath at $37^{\circ} \mathrm{C}$ for one hour, and then subjected to high-speed centrifugation at 2,000 rpm for 20 minutes. Serum samples were drawn for direct identification or kept at $-20^{\circ} \mathrm{C}$. Antibody titers were measured by enzyme-linked immunosorbent assay (ELISA). The enzymelinked reaction plates were coated with Nogo-13 antigenic polypeptide fragments $(0.2 \mu \mathrm{g} / 0.1 \mathrm{~mL}$ per hole $)$ and kept in carbonate buffer (pH 9.6) at $4^{\circ} \mathrm{C}$ overnight. Subsequently, the plates were washed four times with phosphate-buffered saline-Tween (0.01 M phosphate-buffered saline, $\mathrm{pH} 7.4$, containing $0.5 \%$ Tween-20) and dried. The wells were blocked with $1 \%$ bovine serum albumin in carbonate buffer $(\mathrm{pH} 9.6)$ at $37^{\circ} \mathrm{C}$ for one hour. Antisera were added into these holes with seven-fold dilution $(1: 20 ; 1: 40 ; 1: 80 ; 1: 160 ; 1: 320 ; 1: 640$; $1: 1,280)$ at $37^{\circ} \mathrm{C}$ for 30 minutes. The serum of a nonimmunized rat served as the negative control, and bovine serum albumin as the blank control. Horseradish peroxidase-labeled rabbit anti-rat IgG was selected as the secondary antibody. The tetramethyl benzidine aniline method for coloration was performed in this study. Optical density (OD) values were measured at a wavelength of $450 \mathrm{~nm}$ using an ELISA analyzer (EMax Endpoint ELISA reader, Molecular Devices, Sunnyvale CA, USA). 


\section{Safety evaluation of vaccine}

The incidence of experimental autoimmune encephalomyelitis was identified and quantified using a modified version of the Kono standard. ${ }^{16}$ The Kono scoring system is as follows: zero score, no abnormality; 1 , tail paralysis; 2 , hind limb weakness; 3, hind limb paralysis; 4, hind limb and forelimb paralysis or cannot turn over; and 5, death of animal. Meanwhile, the rats were monitored for changes in eating behavior, activity, and weight after immunization.

\section{Isolation, culture, and purification of OEGs}

Isolation of OEGs from the olfactory bulb nerve layer has been described previously by Yan et al. ${ }^{17}$ DF-10S culture medium supplemented with L-glutamine $20 \mathrm{mmol} / \mathrm{L}$ and penicillin/streptomycin $10,000 \mu \mathrm{g} / \mathrm{mL}$ was added and the cells were passed through a $70 \mu \mathrm{m}$ mesh filter. The cell suspension was diluted to $1 \times 10^{6}$ cells $/ \mathrm{mL}$ and plated onto a $25 \mathrm{~cm}^{2}$ flask pretreated with poly-D-lysine $0.2 \mathrm{mg} / \mathrm{mL}$. The cells were incubated for 7 days at $37^{\circ} \mathrm{C}$ with $5 \% \mathrm{CO}_{2}$, with the culture medium changed every 2 days. Six days following plating, the OEGs were purified from contaminated cells using serum-free medium instead of DF-10S medium. The serum-free medium consisted of Dulbecco's Modified Eagle's Medium, biotin $10 \mathrm{ng} / \mathrm{mL}$, insulin $5 \mu \mathrm{g} / \mathrm{mL}$, transferrin $50 \mu \mathrm{g} / \mathrm{mL}$, sodium selenate $5.2 \mathrm{ng} / \mathrm{mL}$, hydrocortisone $10 \mathrm{ng} /$ $\mathrm{mL}$, and glutamine $292 \mathrm{mg} / \mathrm{L}$. Next, the cells were incubated at $3{ }^{\circ} \mathrm{C}$ with $5 \% \mathrm{CO}_{2}$ with a change of culture medium every 3 days. Before implantation, the OEGs were labeled using Hoechst dye.

\section{Experimental design of in vivo study}

Eighty rats were randomly divided into four groups as follows: a vaccine + OEG group (group $A, n=20$ ); a vaccine only group (group $\mathrm{B}, \mathrm{n}=20$ ); an $\mathrm{OEG}$ only group (group $\mathrm{C}$, $n=20)$; and an untreated SCI group ( $n=20$, group D).

\section{Surgical preparation of $\mathrm{SCl}$ model}

In brief, the animals were anesthetized and underwent laminectomy at T9-T10, after which they were subject to a spinal cord contusion using a New York University impactor (W.M. Keck Center for Collaborative Neuroscience, Piscataway, NJ, USA) (10g, 25mm; Figure $1 \mathrm{~A}$ and B). ${ }^{18}$ Group A rats immunized with NogoA vaccine underwent OEG implantation at the time of injury. OEGs labeled with Hoechst dye was carefully injected into the middle of the spinal cord at a $1 \mathrm{~mm}$ distance both proximal and distal from the edges of the lesioned area. From ventral to dorsal, the cells were injected into the following four sites of each cord stump: the ventral funiculus, the gray commissure, the dorsal corticospinal tract, and the fasciculus gracilis. The four injection sites were $1.75 \mathrm{~mm}, 1.25 \mathrm{~mm}, 1 \mathrm{~mm}$, and $0.5 \mathrm{~mm}$ deep, respectively. Each site received $1 \mu \mathrm{L}$ of suspension containing $5 \times 10^{4}$ cells, ie, a total of $2 \times 10^{5}$ cells was injected into each cord stump. Group B comprised immunized rats with SCI contusion. In group $\mathrm{C}$, OEGs were transplanted into the injured spinal cords of the nonimmunized rats. In group D, SCI contusion was created in nonimmunized rats. For consistency, all rats were kept under the same conditions.

\section{Behavioral testing}

Locomotor function on the BBB scale ${ }^{7}$ was evaluated before surgery, then at 2 days and at 2, 4, 6, 8, 10, and 12 weeks postoperatively. The final score for each rat was the mean score given by two examiners.

\section{BDA tracing}

Antegrade biotinylated dextran amine (BDA) tracing was performed for all the rats 12 weeks after surgery. The rats were anesthetized and then fixed onto a stereotactic frame for laminectomy at T5-T6. Using a microscope, the dural sac was cut and the spinal cord was exposed at T5-T6. A 10\% BDA solution dissolved in phosphate buffer

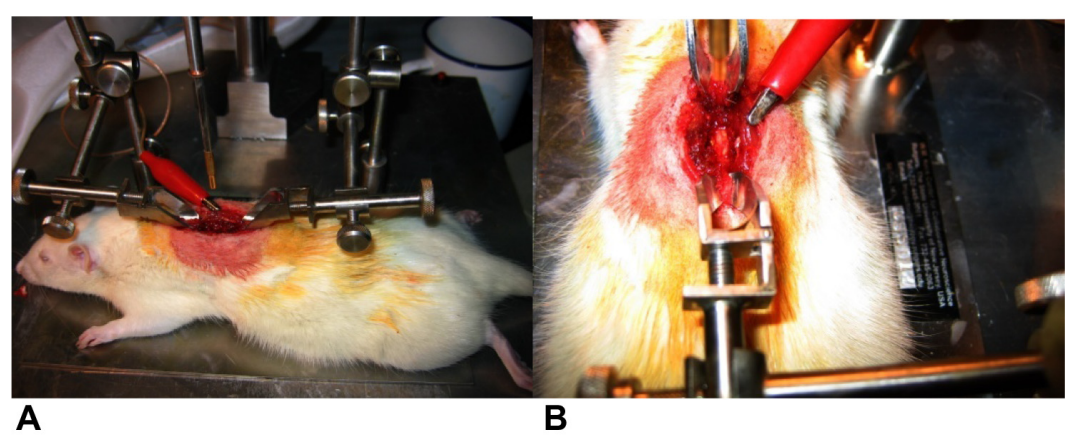

Figure I (A and B) spinal cord contusion injury model made with a New York University impactor. Note: New York University impactor; W.M. Keck Center for Collaborative Neuroscience, Piscataway, NJ, USA. 
(0.1 M, pH 7.2) was injected bilaterally into this location in the corticospinal tract under pressure with a microsyringe (Figure 2). After the injection, the microsyringe was left in place for 5 minutes to ensure that the injected BDA was absorbed into the tissue. The incision was then closed. The rats were euthanized 5 days after the antegrade tracing study.

\section{BDA staining and counting of traced corticospinal tract axons}

BDA staining was performed according to a method described elsewhere. ${ }^{19}$ The spinal cords were quickly removed and placed in fixative at $4^{\circ} \mathrm{C}$ for 4 hours. Frozen sections (30 $\mu \mathrm{m}$ in thickness) were cut and collected in phosphate buffer in a free-floating state. First, the tissue section was placed on a slide in $0.01 \mathrm{M}$ phosphate-buffered saline containing 30\% glycerol. The slides were then observed for axon growth under a fluorescence microscope with blue light excitation, and the number of nerve fibers in the injured area was counted. Second, the slides were dried in an incubator at $38^{\circ} \mathrm{C}$ for one hour and washed twice for 10 minutes in $50 \mathrm{mM}$ Tris-buffered saline ( $\mathrm{pH} 7.4$ ), followed by washing with Tris-buffered saline containing $0.5 \%$ Triton X-100. The slides were then incubated overnight with avidin-biotinperoxidase complex in Tris-buffered saline and Triton X-100 according to the manufacturer's instructions. Thereafter, the 3,3'-diaminobenzidine (DAB) reaction was performed using the Vector DAB kit (Vector Laboratories, Burlingame, CA, USA). The reaction was monitored and terminated by extensive washing in water. The slides were dehydrated and coverslipped for observation. Quantification of the total number of traced corticospinal tract axons cephalic and caudal to the

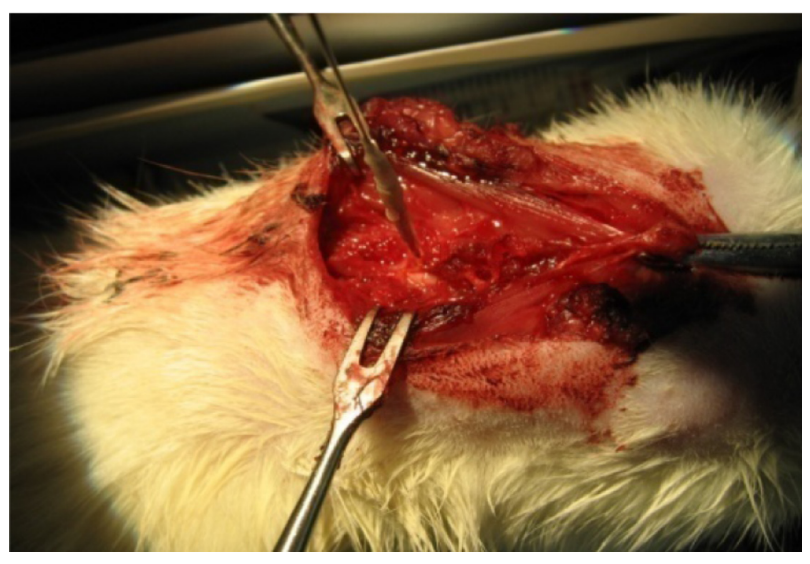

Figure 2 Biotinylated dextran amine tracer being injected into the corticospinal tract in the proximal part of the injured area with a microinjector under the microscope. injured area was performed on cross-sections using light or fluorescence microscopy. Pictures were taken using a digital camera mounted on a microscope. Image analysis software (a computer-assisted digital image analysis system, MPIAS-500 [QingPing Image Engineering Company, Shanghai, People's Republic of China]) was used to assist counting of the traced axons, and by manually setting the relative OD threshold to identify positive staining within a defined target area.

\section{Observation of Hoechst-labeled OEG}

Tissue sections was observed under the fluorescence microscope (wavelength of $365 \mathrm{~nm}$ ultraviolet excitation) to analyze OEG survival, distribution, and migration.

\section{Staining with hematoxylin and eosin}

Hematoxylin and eosin staining was performed to observe the general structure and morphology of the SCI area. Lesion size was determined from a series of cross-sections of a spinal segment taken from T8-T10. Pathologic changes in the area of SCI were observed.

\section{Immunohistochemistry}

The spinal cord was cut at the contused level and fixed in a $10 \%$ formalin solution for 2 hours, then embedded in paraffin and cut into $4 \mu \mathrm{m}$ sections. Primary antibodies (mouse anti-neurofilament SMI3 1, 1:1,000; Sternberger Monoclonals Inc, Baltimore, MD, USA) raised against neurofilament (NF) were diluted in 1\% normal serum, applied to the tissue sections at $37^{\circ} \mathrm{C}$ for one hour, and then at $4{ }^{\circ} \mathrm{C}$ overnight. Sections were incubated with an appropriate biotinylated secondary antibody (1:200; Vector Laboratories) followed by $\mathrm{ABC}$ complex for 45 minutes at room temperature. Diaminobenzidine (brown colored, Sigma Chemical Co, St Louis, MO, USA) was used as the chromatogen. The sections were counterstained with hematoxylin, dehydrated in graded alcohols, and mounted. Sections stained with normal serum were used for the control. Quantification of the total number of positively stained nerve fibers was done using MPIAS.

\section{Statistical analysis}

Statistical processing and analysis were done using Statistical Package for the Social Sciences version 13 software (SPSS Inc, Chicago, IL, USA). The relevant data are reported as the mean \pm standard deviation. Data sets were analyzed for significance using one-way and repeated-measures analysis of variance or simple contrast tests. A $P$-value $<0.05$ was regarded as being statistically significant. 


\section{Results}

\section{Evaluation of Nogo vaccine}

Efficacy evaluation: ELISA test of antibody titers

The serum IgG antibody against NogoA-13 in group $\alpha$ rats was detected by ELISA. After double dilution, serum OD values showed a regular gradient. The antibody against NogoA-13 was not present in rat serum from groups $\beta$ and $\gamma$ (OD value $<0.1$ ). Further, there was no gradient for OD values after double dilution of serum from groups $\beta$ and $\gamma$, the OD curves of which nearly overlapped. There were significant differences in OD values between group $\alpha$ and groups $\beta$ and $\gamma$ by analysis of variance $(P<0.05)$. Differences in OD values between group $\beta$ and group $\gamma$ were not statistically significant $(P>0.05)$. Differences in OD values between groups $\beta$ and $\gamma$ and the nonimmunized negative control group were not statistically significant either (Figure 3).

\section{Safety evaluation of vaccine: incidence}

\section{of experimental autoimmune encephalomyelitis}

Rats in all three groups $(\alpha, \beta, \gamma)$ were less active and ate less on the day following injection of the vaccine, but returned to their normal state by the end of the first day after vaccination. Their body weight gradually increased, and there were no signs suggestive of experimental autoimmune encephalomyelitis, such as weight loss, tail paralysis, hind limb weakness and paralysis, forelimb paralysis, or death. Kono scores, incidence of experimental autoimmune encephalomyelitis, and mortality rate were all zero at 2, 4, 6, and 8 weeks after the first immunization.

\section{Recovery of locomotor function}

After anesthesia, the mean BBB score was zero for all rats. Mean BBB scores in groups A and B reached more than 7 points 2 weeks after surgery. However, other groups scored

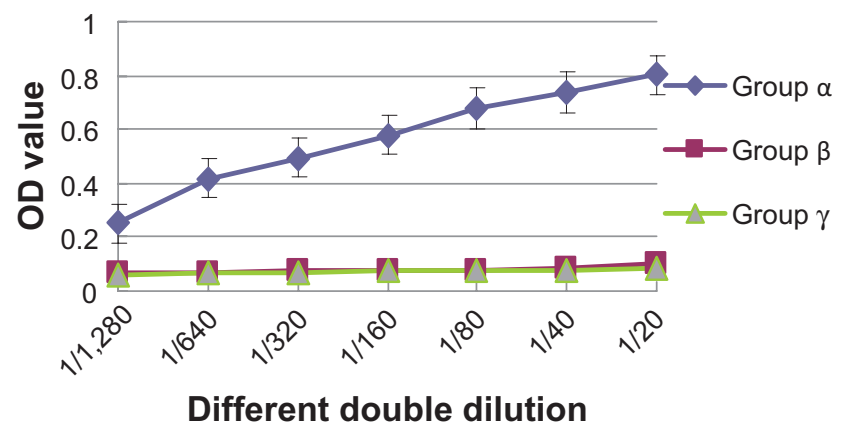

Figure 3 Antibody titer standard curve for groups $\alpha, \beta$, and $\gamma$.

Notes: Group $\alpha$ received a NogoA vaccine immunization; group $\beta$ received complete Freund's adjuvant and incomplete Freund's adjuvant immunization; group $\gamma$ received KLH immunization.

Abbreviations: OD, optical density; KLH, keyhole limpet hemocyanin. less than 7 points. Four weeks after surgery, the mean score recovered to more than 7 points except in group D. Six weeks after surgery, scores in groups A and B increased gradually and were significantly higher than in groups $C$ and $D$. The BBB scores for the four groups at the different time points are shown in Figure 4.

By 12 weeks after surgery, there were significant differences between the four groups ( $F=60.89$, analysis of variance, $P<0.001)$. Groups $\mathrm{A}$ and $\mathrm{B}$ had higher $\mathrm{BBB}$ scores than groups $\mathrm{C}$ and $\mathrm{D}$. The $\mathrm{BBB}$ score in group $\mathrm{A}$ was higher than in group $\mathrm{B}(P=0.038)$, while the $\mathrm{BBB}$ score in group $\mathrm{C}$ was higher than in group $\mathrm{D}(P=0.021)$.

\section{General morphologic observation of spinal cord specimens}

Whole spinal cords were removed from the rats' spinal canals after formaldehyde perfusion at 12 weeks after surgery. The injury areas in groups A and B were smaller than those in groups $\mathrm{C}$ and $\mathrm{D}$. The diameters of the injured spinal cord levels in groups A and B were wider than those in groups $\mathrm{C}$ and D (Figure 5).

\section{Histologic observation}

\section{Analysis of lesion size by hematoxylin}

and eosin staining

Groups A and B had smaller lesion sizes than groups $\mathrm{C}$ and D. The lesion size in group A was the smallest of all the groups. Group D had the largest lesion size and cavity in the SCI zone (Figure 6).

\section{OEG survival and migratory properties}

Under fluorescence microscopy with ultraviolet excitation (365 nm), many fluorescent particles at the cell injection sites and injured areas were seen in the slides. This indicates that a

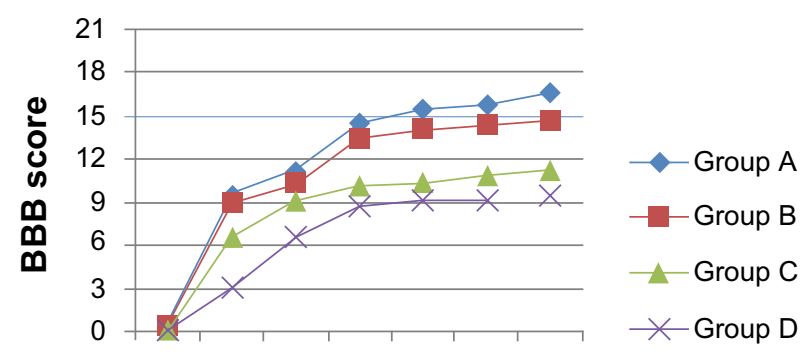

2 d 2 w 4 w 6 w 8 w 10 w 12 w

Time after injury

Figure 4 Curve of BBB score.

Notes: Group A, vaccine + OEG group; group B, vaccine only group; group C, OEG only group; group $\mathrm{D}$, untreated $\mathrm{SCl}$ group.

Abbreviations: BBB, Basso, Beattie, Bresnahan; d, day; w, week; OEG, olfactory ensheathing glial cell; $\mathrm{SCl}$, spinal cord injury. 


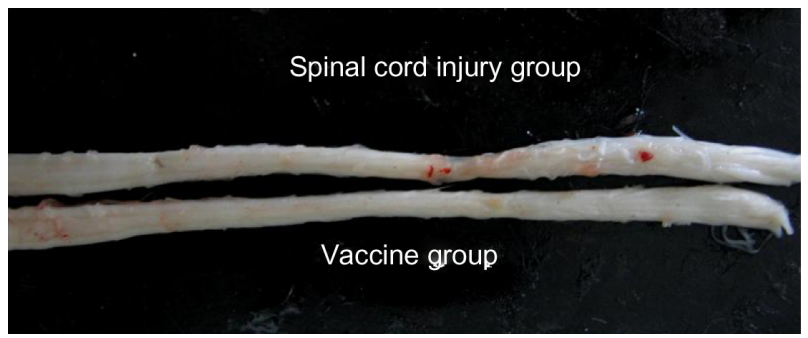

Figure 5 General view of entire spinal cord specimen at 12 weeks after spinal cord injury. The reactive injury area in the control group was larger than in the vaccine group.

large number of OEGs had survived and migrated to the lesion area. As the distance from the injection site increased, the number of surviving OEGs became smaller. Hoechst-labeled OEGs disappeared almost completely at $1.5 \mathrm{~cm}$ from the injection site (Figure 7). There were no significant differences in OEG migration distance between groups $\mathrm{A}$ and $\mathrm{C}$.

\section{Axonal survival and regeneration}

Both dorsomedial corticospinal tracts were labeled with BDA. Regardless of whether a dark field or light field was used for observation, the total number of traced axons cephalic or caudal to the SCI area was greater in groups A and $\mathrm{B}$ than in groups $\mathrm{C}$ and $\mathrm{D}$. The number of labeled axons cephalic or caudal to the area of SCI was greatest in group A and smallest in group D. These differences in axonal survival were statistically significant $(P<0.05)$. NF immunoreactivity at 12 weeks post-injury showed that more NF-positive axons survived in group A. Counting by image analysis software showed higher numbers of neurofibers labeled by NF staining cephalic or caudal to the area of SCI in groups A and B than in groups $\mathrm{C}$ and $\mathrm{D}$ (Figure $8 \mathrm{~A}-\mathrm{D})$.

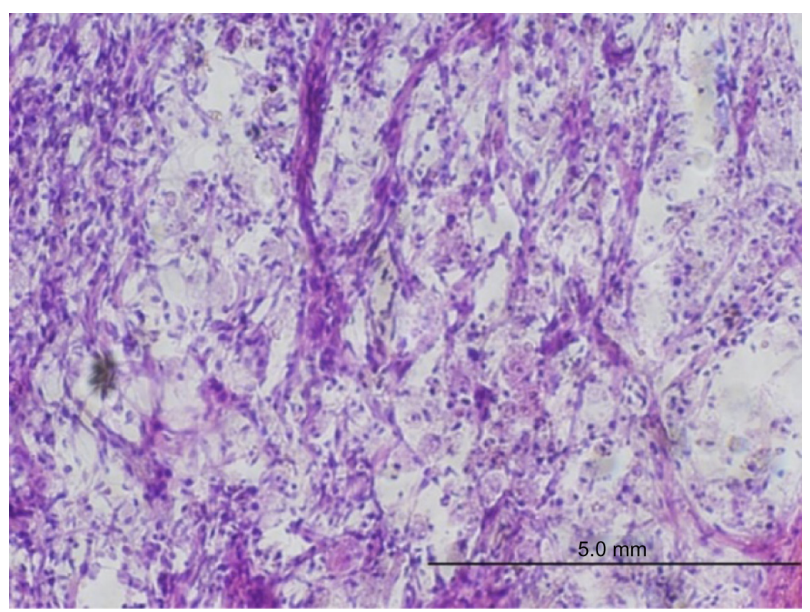

Figure 6 Hematoxylin and eosin staining of injured area in group D (untreated spinal cord injury). There were big cavities and structure disturbance in the injured area of the spinal cord, $100 \times$.

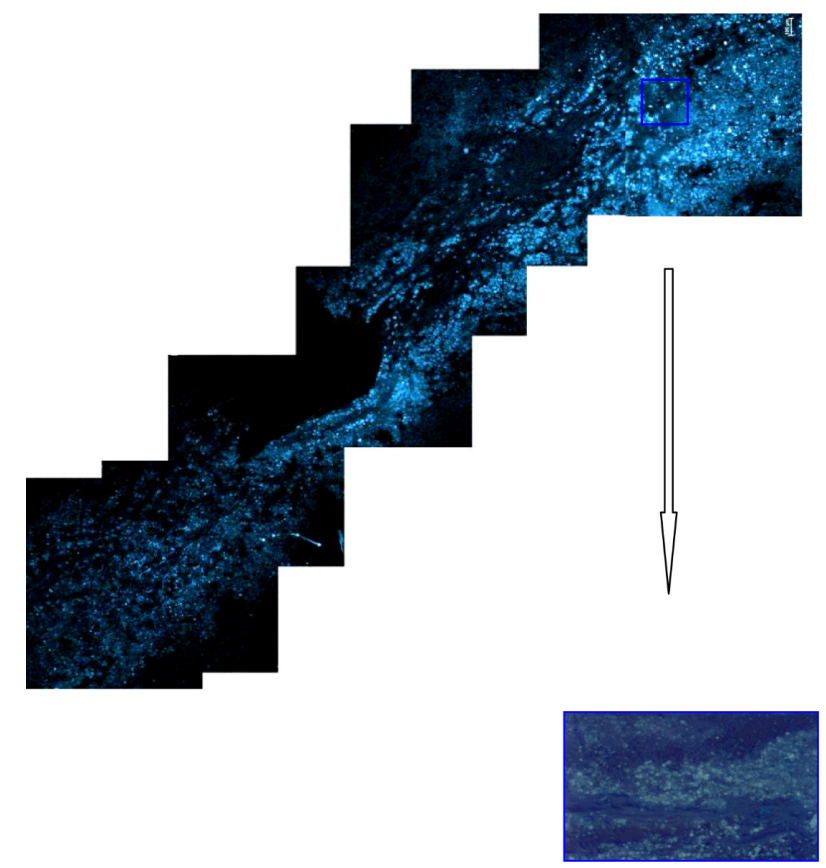

Figure 7 Consecutive views under fluorescence microscope were stitched to observe the survival and migration of the OEGs. The magnified image shows many surviving OEGs migrated $1.5 \mathrm{~cm}$ from the injection site on view of fluorescence microscope. Surviving OEGs were found in the transplanted area. Abbreviation: OEG, olfactory ensheathing glial cells.

\section{Discussion}

An important reason for the extremely limited regenerative capacity of the adult mammalian CNS is the high number of factors inhibiting neuronal regeneration in the CNS. Nogo is one of these inhibitory factors. Nogo-66 is the major molecular functional domain of Nogo exerting such inhibitory effects. Previous studies have shown that a combination of Nogo-66 and NgR (the Nogo-66 receptor on the surface of the neuron) may inhibit neuronal growth and regeneration. ${ }^{13}$ Antibodies ${ }^{20}$ and a competitive antagonist peptide of Nogo-66 may interrupt combination of Nogo-66 and $\mathrm{NgR}$ to promote axonal regeneration of damaged neurons. ${ }^{21}$ These findings have provided new ideas and therapeutic targets for research on neurologic regeneration after SCI.

\section{Feasibility of vaccine treatment in $\mathrm{SCl}$}

It was previously considered that the inflammatory response is harmful during repair of the CNS. A study by Fitch and Silver ${ }^{10}$ found that the inflammatory process triggered a cascade reaction secondary to CNS injury, ending with a cavity and glial scar in the area of SCI. This point is reinforced by the neuroprotective effects of high-dose methylprednisolone. However, recent research ${ }^{21}$ has found that SCI stimulates the autoimmune response, which may protect neurons from further damage, ie, some immune responses are necessary 
A

B
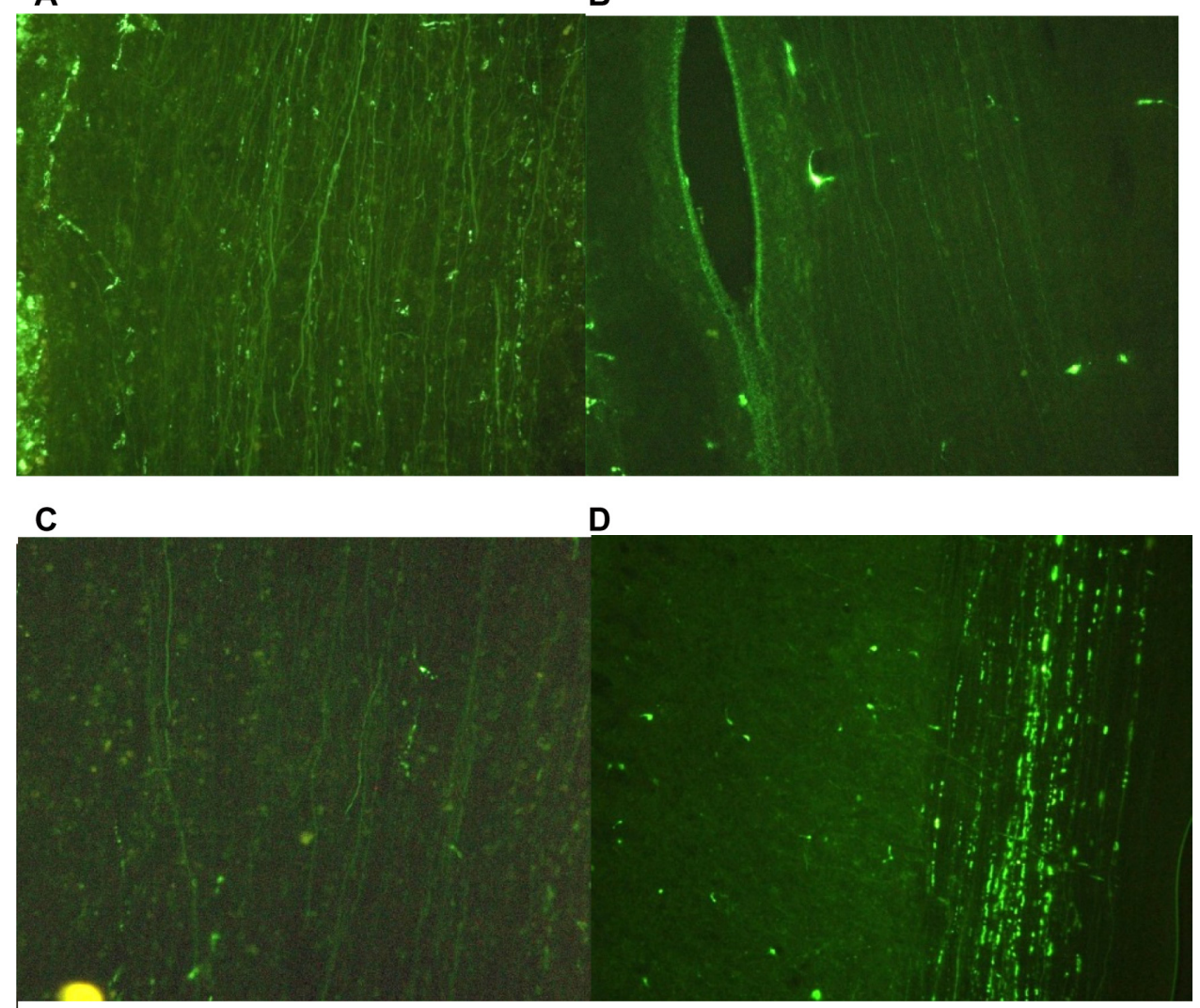

Figure 8 Fluorescence microscopic images showing nerve fibers in the distal spinal cord below the injured level in dark field. (A) Distal nerve fibers in group A, I00X. (B) Distal nerve fibers in group B, 100x. (C) Distal nerve fibers in group C, 100×. (D) Distal nerve fibers in group D, 100×.

Notes: Group A, vaccine + OEG group; group B, vaccine only group; group C, OEG only group; group D, untreated SCI group.

Abbreviations: OEG, olfactory ensheathing glial cell; SCl, spinal cord injury.

for repair and homeostasis in the CNS. Perhaps immune responses under strict regulation are beneficial for repair of spinal cord injury and improvement of neurologic deficits.

In certain pathologic conditions, such as $\mathrm{SCI}$, immune cells or antibodies can enter the damaged area of the spinal cord and have a marked effect if the blood-spinal cord barrier is damaged. ${ }^{22}$ Jensen et $\mathrm{al}^{14}$ reported finding that the bloodspinal cord barrier was destroyed and opened not only in the immediate damaged area but also in the area peripheral to the damaged area. That may be because of activation of microglia and/or macrophages. Such selective opening of the blood-spinal cord barrier in a region adjacent to the damaged spinal cord could be beneficial for entry of antibodies into the area of SCI. This prospect provides the impetus for further research and development of an SCI vaccine.

\section{Safety of polypeptide vaccine}

Peptides are short protein sequences. Peptide vaccines use only part of an organism to spark an immune response. Animals can be immunized with a single protective antigen, or even a single major antigenic epitope instead of a complete pathogen vaccine. This kind of vaccine may induce immune responses against invasive pathogens and avoid pathologic immune reactions from a similar antigen in normal body tissues and pathogenic microorganisms. A polypeptide as a vaccine should not only be closely related to Nogo-66 activity but also contain epitopes stimulating antibody production. Considering the risk of experimental autoimmune encephalomyelitis resulting from an autoimmune response against the normal brain and spinal cord with whole sequence NogoA or Nogo-66, ${ }^{23}$ the vaccine was made using 13 peptide fragments from Nogo-66 as an antigenic determinant to immunize rats and reduce cross-immunopathologic responses. In this study, as expected, rats immunized with the vaccine made from Nogo-13 peptide were less active and ate less on the day following immunization. However, the rats returned to their normal state after only one day. This may have resulted from the immune response against the vaccine. Body weight gradually increased in the immunized animals. There were no signs of experimental autoimmune encephalomyelitis, such as weight loss, tail paralysis, hind limb weakness and paralysis, forelimb paralysis, or death. 
The Kono score, incidence of experimental autoimmune encephalomyelitis, and mortality rate were all zero in this study. In general, the safety of this vaccine is confirmed by our preliminary observations.

\section{Immunogenicity enhancement of polypeptide}

Immunization with the free synthetic peptide generally caused a moderate-intensity immune response. Small molecular peptide antigens can be attached to a macromolecular carrier as polypeptide vaccines. After formation of the chimeric polypeptide vector, the animals in our study were immunized with the chimeric vaccine. The vaccine was recognized and dealt with by mononuclear macrophages and other cells delivering the antigen to T-lymphocytes and B-lymphocytes. The antigenic polypeptide was made up of 13 amino acids and coupled with KLH. One KLH molecule may combine with many polypeptides to enhance the immunogenicity of a vaccine. The antibody against Nogo-13 was detected with ELISA in the serum of all the immunized rats. These animals were selected for further study. After double dilution, the antibody level in serum showed a regular gradient. The results of ELISA identified the antigen-antibody reaction, confirming the efficacy of the Nogo-13 polypeptide vaccine.

\section{Balance of supporting and inhibiting factors OEG as a supportive factor}

Transplanted cells may serve as a vehicle for infusion of survival and growth factors and, in addition, provide a source of molecules that counteract the inhibition caused by glial cells, and become the main supportive factor for repair of SCI. So far, a variety of cell types have been grafted into SCI tissue for regeneration of axons in the inhospitable CNS environment. The olfactory nerve can regenerate in all mammalian. However, the CNS cannot do this. In the olfactory nerve system, OEGs are wrapped around olfactory nerve fibers to prevent these fibers coming into contact with other glial cells in the CNS pathway. That means that OEGs may prevent olfactory axons from coming into contact with the inhibitory CNS environment and provide axons with the appropriate conditions for regeneration and elongation. OEGs can secrete neurotrophic factors, adhesion molecules, and neurogenesis factors. Previous research has shown that OEGs are capable of axon remyelinization and migration in the spinal cord. In conclusion, OEG transplantation promotes histologic repair and functional improvement of the injured mammalian spinal cord.
The present study also confirmed the results of previous research. A large number of circular fluorescent substances in the nuclei of OEGs marked with Hoechst dye were found in the injured area of the spinal cord 3 months after OEG transplantation. This means that most of the transplanted OEGs survived in the spinal cord. Another remarkable finding was the migration of OEG from the injected site to the injured area. There were no significant differences in migratory distance between group A and group $\mathrm{C}$, ie, the migratory distance of the OEGs did not change obviously under the influence of the antibody against Nogo. However, the BBB score in group A (vaccine + OEG group) was higher than in group B (vaccine). In addition, the $\mathrm{BBB}$ score in group $\mathrm{C}(\mathrm{OEG})$ was better than in group D. The results of BDA tracing and NF staining also confirmed that OEG transplantation were beneficial for promotion of nerve fiber regeneration. The nerve fiber counts in group $\mathrm{A}$ in the proximal and distal areas were higher than group B. Furthermore, group C counts were higher than group D. All these findings indicate the OEGs may play a very important role in the repair and regeneration of nerves in SCI.

\section{Antagonism of inhibitory factors in repair of SCl: neuroprotective effects of Nogo vaccine}

Previous studies have reported that the antibody for Nogo, termed IN-1, allows axons to grow a relatively long distance in the rat SCI model. This antibody increases the plasticity of neurologic structures in the spinal cord, and is beneficial for functional recovery. ${ }^{24}$ In addition, IN-1 has synergism with NT-3 and brain-derived neurotrophic factor. However, the antibody has the problem of a short half-life. To prevent cross immune reactivity with full-length Nogo-66 polypeptide vaccine, leading to impairment of normal autologous nerve structures, the Nogo-13 polypeptide was synthesized and coupled with KLH to prepare the protein vaccine. The rats were immunized with this Nogo-13 vaccine by injection into the peritoneal cavity. This enabled the rats to produce the antibody against NogoA-13 before SCI. The antibody against the polypeptide was identified in an in vitro study using the antigen-antibody binding reaction method. When SCI occurred, the antibody could block the inhibitory effects of Nogo through the damaged blood-spinal cord barrier. The greatest benefit of vaccine treatment was immediate, ie, early antagonism of inhibitory factors once the blood-spinal cord barrier was opened as a result of SCI. According to the emergency hierarchy of different countries, the time point of treatment usually was different. The vaccine had special clinical value as a "first-time treatment".

In this study, we found that the BBB score in the vaccine group was higher than in the other groups 2 days after 
SCI. Recovery of locomotor function was also faster than in the other groups at 2 weeks after SCI. The reason for this interesting finding may lie in the fact that the antibodies and immune cells activated by the Nogo vaccine play a role in relieving secondary SCI. Depending on the area of injury in the spinal cord specimens, the scar and diameter of the injured level in group A (vaccine + OEG) and group B (vaccine) were shorter and larger than in group $C$ (OEG) and group D (injured group), respectively. The hematoxylin and eosin staining results for the injured area confirmed this observation. Cell numbers in groups A and B were greater than in groups $\mathrm{C}$ and D. Some of these cells were inflammatory cells, such as macrophages or immune cells activated by the vaccine. When SCI occurred, a large amount of Nogo protein was released into the damaged area, with destruction of the blood-spinal cord barrier. Immune cells activated by the vaccine may migrate into the SCI tissue.

Not all inflammatory responses are necessarily harmful to the SCI. Previous studies have shown that macrophages can help to remove local cell debris from the SCI area, and are beneficial for regeneration of damaged neurologic structures. The BBB score in group A was lower than in group B at 2 days after injury, possibly because of microtrauma arising from the cell injection procedure. Long-term restoration of neurologic function relied mainly on regeneration of axons. Therefore, the BBB score of group A gradually increased, and became higher than in group $\mathrm{B}$.

In addition, the BBB score and nerve fiber counts in the BDA test were higher in group B than in group D. NF-positive axons surviving on BDA tracing distal to the damaged area were significantly greater in number than in group D. This result considered that the inhibition effects of NogoA could be neutralized by antibodies generated from Nogo13 vaccine to promote axonal regeneration.

The results for group B were better than in group $C$ for all aspects of the BBB score, including macroscopic structures in the spinal cord, surviving NF-positive axons, and BDAlabeled nerve fiber counts. We suspect that there are two possible reasons for this. First, the antibodies and immune cells stimulated by this vaccine might reduce secondary SCI. Second, antagonism of regenerative inhibitory factors might play a more important role in the repair of SCI.

\section{Effects of Nogo vaccine combined with OEG transplantation in repair of $\mathrm{SCl}$ : neurologic function recovery and histologic changes}

The BDA tracing results showed that the number of labeled nerve fibers in group A in the area of SCI within $5 \mathrm{~mm}$ was greater than in the other three groups $(P<0.01)$. As seen from this experiment, surviving axons could be found from the proximal to central injured zone of the spinal cord in all four groups. However, the greatest number of surviving axons was seen in the combination treatment group. The above results also show that the axons had some capacity to regenerate even without any therapeutic measures. However, only a very small number of nerve fibers regenerated. At the same time, this experiment also confirmed that OEGs have the ability to promote regeneration of axons in SCI. On the caudal side of the SCI area, there were some BDAlabeled axons through the damage zone from proximal to caudal, although the number was significantly less than in the rostral area of the contused spinal cord. The most probable reason for this was that the glial scar blocked the passage of axons. Of course, because of the limitation of this SCI model, it was not confirmed that the surviving axons were regenerated ones crossing the injured area, and this needs further study.

According to our experimental results, the BBB score in group A was greater than in the other groups, meaning that augmentation of regenerative contributing factors and antagonism of barriers to regeneration can significantly promote functional recovery from SCI.

\section{Disclosure}

The authors report no conflicts of interest in this work.

\section{References}

1. Benfey M, Aguayo AJ. Extensive elongation of axons from rat brain into peripheral nerve grafts. Nature. 1982;296:150-152.

2. Horner PL, Gage FH. Regenerating the damaged central nervous system. Nature. 2000;407:963-970.

3. Hernández J, Torres-Espín A, Navarro X. Adult stem cell transplants for spinal cord injury repair: current state in preclinical research. Curr Stem Cell Res Ther. 2011;6:273-287.

4. Blesch A, Fischer I, Tuszynski MH. Gene therapy, neurotrophic factors and spinal cord regeneration. Handb Clin Neurol. 2012;109: 563-574.

5. Raisman G, Barnett SC, Ramón-Cueto A. Repair of central nervous system lesions by transplantation of olfactory ensheathing cells. Handb Clin Neurol. 2012;109:541-549.

6. Wu J, Sun TS, Ren JX, et al. Ex vivo non-viral vector-mediated neurotrophin-3 gene transfer to olfactory ensheathing glia: effects on axonal regeneration and functional recovery after implantation in rats with spinal cord injury. Neurosci Bull. 2008;24:57-65.

7. Basso DM, Beattie MS, Bresnahan JC. A sensitive and reliable locomotor rating scale for open field testing in rats. J Neurotrauma. 1995;12:1-21.

8. Huber AB, Schwab ME. Nogo-A, a potent inhibitor of neurite outgrowth and regeneration. Biol Chem. 2000;381:407-419.

9. Xie F, Zheng B. White matter inhibitors in CNS axon regeneration failure. Exp Neurol. 2008;209:302-312.

10. Fitch MT, Silver J. CNS injury, glial scars, and inflammation: inhibitory extracellular matrices and regeneration failure. Exp Neurol. 2008;209: 294-301. 
11. Bourquin $\mathrm{C}$, van der Haar ME, Anz D, et al. DNA vaccination efficiently induces antibodies to Nogo-A and does not exacerbate experimental autoimmune encephalomyelitis. Eur J Pharmacol. 2008;588:99-105.

12. Caroni P, Schwab ME. Antibody against myelin-associated inhibitor of neurite growth neutralizes nonpermissive substrate properties of CNS white matter. Neuron. 1988;1:85-96.

13. Foumier AE, Grandpre T, Strittmatter SM. Identification of a receptor mediating Nogo-66 inhibition of axonal regeneration. Nature. 2001;409:341-346.

14. Jensen MB, Finsen B, Zimmer J. Morphological and immunophenotypic microglial changes in the denervated fascia dentata of adult rats: correlation with blood-brain barrier damage and astroglial reactions. Exp Neurol. 1997;143:103-116.

15. Huang DW, McKerracher L, Braun PE, et al. A therapeutic vaccine approach to stimulate axon regeneration in the adult mammalian spinal cord. Neuron. 1999;24:639-647.

16. Kono DH, Urban JL, Horvath SJ, et al. Two minor determinant of myelin basic protein induce experimental allergic encephalomyelitis in SJL/J mice. J Exp Med. 1988;168:213-227.

17. Yan H, Bunge MB, Wood PM, Plant GW. Mitogenic response of adult rat olfactory ensheathing glia to four growth factors. Glia. 2001;33:334-342.
18. Gruner JA. A monitored contusion model of SCI in the rat J Neurotrauma. 1992;9:123-128.

19. Inman DM, Steward O. Ascending sensory, but not other long-tract axons, regenerate into the connective tissue matrix that forms at the site of a spinal cord injury in mice. J Comp Neurol. 2003;462:431-449.

20. Raineteau O, Fouad K, Noth P, et al. Functional switch between motor tracts in the presence of the mAb IN-1 in the adult rat. Proc Natl Acad Sci U S A. 2001;98:6929-6934.

21. Li S, Strittmatter SM. Delayed systemic Nogo-66 receptor antagonist promotes recovery from spinal cord injury. J Neurosci. 2003;23: 4219-4227.

22. Ye JH, Houle JD. Treatment of the chronically injured spinal cord with neurotrophic factors can promote axonal regeneration from supraspinal neurons. Exp Neurol. 1997;143:70-81.

23. Teng FY, Tang BL. Nogo signaling and non-physical injury-induced nervous system pathology. J Neurosci Res. 2005;79:273-278.

24. Hauben E, Butovsky O, Nevo U, et al. Passive or active immunization with myelin basic protein promotes recovery from spinal cord contusion. J Neuroscience. 2000;20:6421-6430.
Journal of Neurorestoratology

\section{Publish your work in this journal}

The Journal of Neurorestoratology is an international, peer-reviewed, open access online journal publishing original research and review articles on the subject of Neurorestoratology. To provide complete coverage of this revolutionary field the Journal of Neurorestoratology will report on relevant experimental research, technological advances, and

\section{Dovepress}

clinical achievements. The manuscript management system is completely online and includes a very quick and fair peer-review system, which is all easy to use. Visit http://www.dovepress.com/testimonials.php to read real quotes from published authors.

Submit your manuscript here: http://www.dovepress.com/journal-of-neurorestoratology-journal 